\title{
Factores asociados con el desarrollo de la investigación en enfermería en el área clínica
}

\author{
Factors associated with the development of research in nursing in the clinical area
}

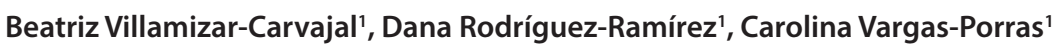

Para citar este artículo: Villamizar-Carvajal B, Rodríguez-Ramírez D, Vargas-Porras C. Factores asociados con el desarrollo de la investigación en enfermería en el área clínica. Ustasalud 2018; 17:-23-33

Licencia Creative Commons \begin{abstract}
(c) (1) (\$) lo tanto, los lectores pueden acceder libremente a los artículos en su formato .pdf, igualmente podrán descargarlos y difundirlos; sin embargo no podrán modificarlos o alterarlos, adicionalmente se debe reconocer la autoría de las personas que figuran en las publicaciones, pero estas no podrán ser comercializadas.
\end{abstract}

\section{RESUMEN}

Objetivo: Determinar los factores asociados con el desarrollo de la investigación en enfermería en el área clínica.

Materiales y métodos: Estudio descriptivo correlacional de corte transversal, muestra conformada por 80 enfermeras asistenciales. La información se recolectó mediante un instrumento de medición previamente validado, se elaboró la correspondiente base de datos en Excel. Se utilizó el paquete estadístico Stata 12 para el procesamiento y análisis de la información; se hizo el análisis descriptivo de los factores sociodemográficos, seguido de la evaluación de la consistencia interna del cuestionario, mediante el cálculo del coeficiente de Cronbach, así mismo, se realizó el análisis factorial exploratorio para establecer la relación entre sus variables, una prueba de $\mathrm{X}^{2} \mathrm{y}$ una rotación de Varimax.

Resultados: Se encontró que, a mayor nivel educativo, mayor motivación para investigar y sentirse capaz de liderar trabajos de investigación. Mejorar el currículum y permitir ser más competitivo. Así mismo, el menor tiempo de antigüedad y estar motivados en el trabajo anima a investigar y alcanzar un grado superior.

Conclusión: Durante las últimas décadas la demanda del conocimiento y la práctica clínica basada en la evidencia ha aumentado y se requiere una colaboración entre enfermería a nivel académico y clínico para impulsar la investigación y que le sea útil a la práctica. Es necesario que las instituciones de salud favorezcan la investigación y se desarrolle una cultura para fortalecer la capacidad de investigación en los profesionales de enfermería clínicos y así, generar resultados que mejoren el cuidado de los pacientes.

Palabras clave: Actitud, motivación, conocimiento, enfermeras, investigación.

\section{ABSTRACT}

Objective: Determine the factors associated with the development of nursing research in the clinical area.

Methods: Cross-sectional descriptive study, consisting of 80 nurses. The information was collected by means of a previously validated measurement instrument, the corresponding database was elaborated in Excel. The software Stata: Release 12 (StataCorp) was used for the processing and analysis of the information; The descriptive analysis of the sociodemographic factors was done, followed by the evaluation of the internal consistency of the questionnaire by calculating the cronbach coefficient, as well as the exploratory factor analysis to establish the relationship between its variables, a test of $\mathrm{chi}^{2}$ and a rotation of Varimax.

Results: The higher the educational level, the more motivated to investigate and feel able to lead research work. Researching improves the curriculum and allows to be more competitive. Likewise, the shorter seniority and being motivated at work encourages research and reaching a higher grade.

Conclusion: During the last decades the demand for knowledge and clinical practice based on evidence has increased and a collaboration between nursing at the academic and clinical levels is required to promote research, that is useful to practice. It is necessary for health institutions to favor research and develop a culture to strengthen the research capacity of clinical nursing professionals and thus generate results that improve patient care.

Keywords: Attitude, motivation, knowledge, nurses, research.
1 Grupo de investigación GRINFER, Escuela de Enfermería, Facultad de Salud, Universidad Industrial de Santander.

Autor de correspondencia:

Carolina Vargas Porras. Profesora Escuela de Enfermería. Facultad de Salud. Universidad Industrial de Santander.

Correo electrónico: carvarpo@uis.edu.co.
Recibido para publicación: 19 de julio 2018. Aceptado para publicación: 14 de octubre 2018. 


\section{INTRODUCCIÓN}

La investigación en enfermería, se define como un "proceso científico que valida y mejora el conocimiento existente y genera nuevo conocimiento que influye en la práctica enfermera, directa o indirectamente" ${ }^{\text {. }}$.

A nivel mundial la investigación en enfermería ha evolucionado desde las primeras investigaciones desarrolladas por Nightingale desde 1850 sobre la interacción existente entre las personas y su entorno; hasta las investigaciones más recientes desarrolladas en los ámbitos educativo, clínico y comunitario, donde han surgido diferentes líneas y grupos de investigación, identificando prioridades cada vez con más apoyo financiero ${ }^{1}$.

La evidencia del avance en investigación en enfermería está dada por el aumento en la producción científica, aunque la mayoría de los trabajos han sido desarrollados por enfermeros de la academia, en comparación con los del área asistencial, quienes están más en contacto con los usuarios y pueden quizás evidenciar de manera más directa las necesidades de fenómenos de estudio para mejorar los cuidados de enfermería; sin embargo, es preocupante el poco aporte del área asistencial a la investigación en enfermería ${ }^{2}$.

Desde el marco legal a nivel internacional y nacional, se establece que el profesional en enfermería tiene como función especial investigar, establecido desde la CIE (Consejo Internacional de Enfermería), para mejorar la calidad de la atención a los pacientes mediante el conocimiento e incorporación de investigación pertinente en la práctica. Así mismo, se sustenta en la ANEC (Asociación Nacional de Enfermeras de Colombia) y en la Ley 266 de $1996^{3}$ la cual reglamenta la profesión de enfermería en Colombia, donde se dicta que el profesional de enfermería debe aportar al trabajo sectorial e intersectorial por medio de la investigación. Sin embargo, el potencial de profesionales de enfermería como productores de investigación no se está desarrollando plenamente ${ }^{4}$.

Los estudios muestran los diversos problemas que tiene la enfermería en la asistencia para el desarrollo de investigación, como lo es la falta de tiempo ${ }^{5}$, dificultades para obtener fondos para investigación, falta de autoridad de los profesionales de enfermería, falta de apoyo ${ }^{6} \mathrm{y}$ falta de conocimiento relacionado con investigación ${ }^{5}$. En una encuesta realizada a profesionales de enfermería sobre las actitudes de investigación; la mayoría refirió que utiliza los hallazgos en su práctica, pero solo un pequeño porcentaje se encontraba realizando un proyecto de investigación en ese momento ${ }^{7}$.

De igual manera, en Australia, en un estudio ${ }^{5}$ sobre actitudes e intereses en la investigación se evidenció que las principales limitaciones de los profesionales de enfermería a la hora de realizar un proyecto de investigación son la falta de tiempo y de conocimientos. Así mismo, en un estudio en China ${ }^{8}$ se encontró que cuando mayor es la preparación educativa más activa es la participación de los profesionales de enfermería en investigación.

Existen pocos estudios relacionados con la capacidad de investigación en los profesionales de enfermería clínicos, porque la mayoría están enfocados en el uso de la investigación o la práctica basada en la evidencia ${ }^{2}$. Por lo tanto, esto sugiere que la investigación no es una cuestión de elección personal; si no que abarca una responsabilidad y es parte de la función de enfermería en la búsqueda de un mayor reconocimiento profesional para mejorar los resultados de la atención al paciente ${ }^{6}$. Como se puede evidenciar diversos países han investigado este aspecto en enfermería, pero en Colombia aún no se evidencian estudios relacionados con esta importante temática. Por lo tanto, el propósito de este estudio fue determinar los factores asociados con el desarrollo de la investigación en enfermería en el área clínica.

\section{MATERIALES Y MÉTODOS}

Estudio descriptivo correlacional de corte transversal, realizado en una institución de salud de tercer nivel en Bucaramanga (Colombia) entre octubre de 2016 hasta mayo de 2017. La población fueron 150 profesionales de enfermería, población total que labora en la institución de salud de tercer nivel. La muestra no probabilística, estuvo conformada por 80 enfermeras asistenciales de diferentes áreas que cumplieron con los siguientes criterios de elegibilidad. Criterios de inclusión: profesionales de enfermería que laboren en la institución de salud y profesionales de enfermería que laboren en las diferentes áreas de desempeño clínico. Criterios de exclusión: se excluyeron a los profesionales de enfermería que se desempeñaran principalmente en el área administrativa. 
Se utilizó como instrumento de medición un cuestionario previamente validado y diseñado para evaluar la motivación hacia la investigación de los profesionales de enfermería . El cuestionario incluye diferentes preguntas para evaluar el grado de actitud, situaciones que motivan, favorecen o dificultan la investigación. Está compuesto por 58 ítems de los cuales: 16 ítems, son datos de tipo socio-demográfico y laboral y, 42 ítems divididos en 5 bloques, evalúan la organización de la vida personal y profesional, la gestión del tiempo, los conocimientos y preparación, los recursos disponibles y apoyos, y el desarrollo profesional y motivación; con una escala de respuestas que va desde totalmente de acuerdo a totalmente en desacuerdo. La recolección de los datos se realizó en cada servicio de la institución, por medio del autodiligenciamiento del cuestionario, en formato físico, garantizándose el anonimato.

La base de datos se elaboró en Microsoft Excel mediante la utilización de códigos. Se utilizó el paquete estadístico Stata 12.0 (StataCorp) para el procesamiento y análisis de la información.

Se hizo el análisis descriptivo de los factores sociodemográficos, seguido de la evaluación de la consistencia interna del cuestionario mediante el cálculo del coeficiente de Cronbach, así mismo, se llevó a cabo el análisis factorial exploratorio para establecer la relación entre sus variables mediante el coeficiente de correlación de Spearman y la prueba $\mathrm{X}^{2} \mathrm{y}$ una rotación de Varimax.

\section{Aspectos éticos y legales}

Se siguieron los lineamientos de la Resolución 008430 del Ministerio de Salud de Colombia ${ }^{10}$, según la cual el presente trabajo se clasifica como una investigación sin riesgo. Esta investigación contó con el aval ético de la Universidad Industrial de Santander. Se tuvieron en cuenta las pautas éticas internacionales para la investigación biomédica en seres humanos recomendadas por CIOMS ${ }^{11}$ y la Declaración de Helsinki. Se hizo consentimiento informado individual en forma verbal y escrita.

\section{RESULTADOS}

En cuanto a las características socio-demográficas, el $100 \%$ de las participantes fueron de género femeni- no, el $45 \%$ solteras, y el $44 \%$ casadas, el promedio de edad de las participantes fue de 31 años, el $79 \%$ refiere convivir con al menos una persona y el $68 \%$ tener personas a su cargo.

Las participantes pertenecen a las diferentes áreas de servicios de hospitalización (Tabla 1).

Tabla 1. Áreas de servicios

\begin{tabular}{lcc}
\hline Servicio o unidad actual & $\mathrm{n}$ & $(\%)$ \\
\hline Pediatría & 13 & $(16)$ \\
\hline UCI & 11 & $(14)$ \\
\hline Medicina interna & 10 & $(13)$ \\
\hline Ginecología & 9 & $(11)$ \\
\hline Urgencias & 6 & $(8)$ \\
\hline Cirugía general & 6 & $(8)$ \\
\hline Especialidades quirúrgicas & 6 & $(8)$ \\
\hline Oncología & 3 & $(4)$ \\
\hline Unidad de quemados & 3 & $(4)$ \\
\hline Calidad & 3 & $(4)$ \\
\hline Quirófano & 3 & $(4)$ \\
\hline Comité de educación & 3 & $(4)$ \\
\hline Hematología & 3 & $(4)$ \\
\hline Central de esterilización & 1 & $(1)$ \\
\hline
\end{tabular}

El rango de antigüedad en el hospital más frecuente fue de 1-5 años que equivale al 55\% de las participantes (Figura 1). En cuanto al tiempo de permanencia en el servicio, el $76 \%$ ha estado trabajando en el mismo servicio en los últimos 6 meses; las demás, han cambiado de servicio al menos una vez. Así mismo, el $69 \%$ tiene un contrato fijo.

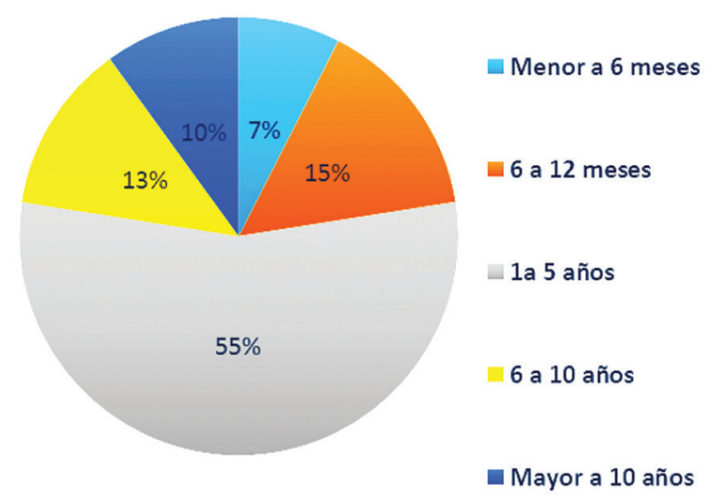

Figura 1. Tiempo de antigüedad en el hospital. 
En cuanto a la formación de las participantes, se evidenció que la mayoría no tiene otros niveles educativos aparte del pregrado y ninguna tiene doctorado (Figura 2). Igualmente, se resalta que el $69 \%$ no tiene ninguna experiencia investigadora (Figura 3) y que el $41 \%$ tuvo formación específica en investigación durante su pregrado y el $29 \%$ durante su posgrado.

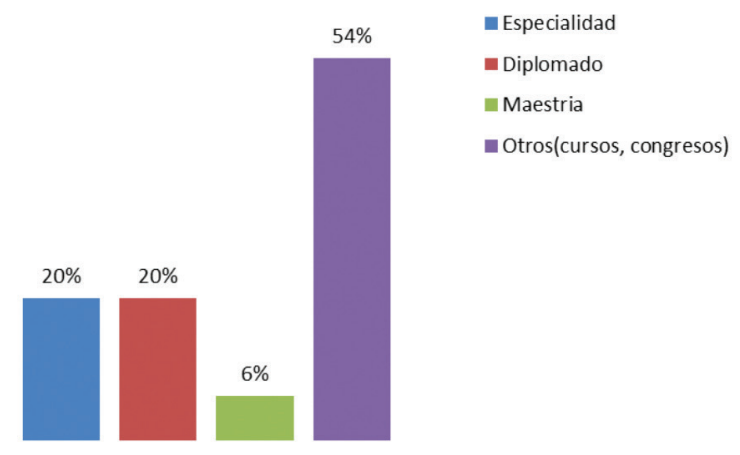

Figura 2. Nivel educativo titulaciones obtenidas.

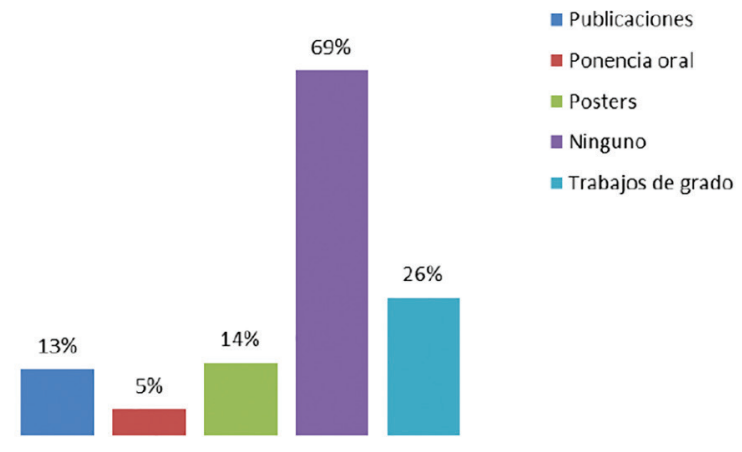

Figura 3. Experiencia investigativa de las participantes.

En relación con los aspectos que tienen que ver con la investigación, las participantes consideran que investigar mejora el desarrollo de la profesión (79\%), permite mejorar la calidad asistencial (69\%), detecta problemas para aportar soluciones $(45 \%)$, requiere de mucho tiempo y esfuerzo (26\%) y lo considera una actividad innecesaria (25\%).

En lo específico para el desarrollo de la investigación de las participantes en cada bloque del cuestionario se observó lo siguiente:
Bloque 1: Organización de la vida personal y profesional: gestión del tiempo

En este bloque se incluyen aspectos que permiten medir cómo influye la vida personal y las cargas laborales en la investigación de enfermería.

$\mathrm{Al}$ 69\% le resulta difícil investigar fuera de su horario laboral, el $41 \%$ considera que sus obligaciones fuera del horario laboral no le suponen un obstáculo para investigar. El 64\% refiere que no dispone de tiempo durante su jornada laboral para investigar. El $43 \%$ piensa que investigar en su horario laboral entorpecería el resto de sus funciones, otro problema importante es la elevada carga asistencial, el 58\% opina que esta carga obstaculiza la investigación durante su jornada laboral. Otra dificultad señalada como impedimento para investigar es la distribución de la jornada laboral por turnos (43\%). El 60\% afirma que se debería emplear parte de la jornada laboral para trabajar en estudios de investigación. El $78 \%$ considera que su vida personal no se verá afectada si hace un trabajo de investigación.

\section{Bloque 2: Conocimientos y preparación}

Este bloque se refiere a los conocimientos que tienen los profesionales de enfermería y si existe suficiente formación en investigación. El 46\% considera que posee suficientes conocimientos para realizar un proyecto de investigación. El 50\% refiere que no existe suficiente formación en investigación y el 79\% considera que se deberían realizar cursos de posgrado relacionados con la materia. Además el $95 \%$ manifiestan que los planes de estudio deberían incluir formación en investigación. La mayoría consideró que, tanto el saber otros idiomas (96\%) como tener conocimientos sobre nuevas tecnologías (95\%) ayudan a la tarea investigadora. Hay que destacar que la gran mayoría (98\%) refiere que los profesionales de enfermería deben leer revistas científicas al menos una vez al mes. Por último, el $89 \%$ considera que el profesional de enfermería está igual de capacitado que otros profesionales sanitarios para realizar un estudio de investigación.

\section{Bloque 3: Recursos disponibles y apoyos}

Los ítems de este bloque pretenden conocer si la investigación de enfermería recibía los mismos apo- 
yos externos, económicos y materiales, que las investigaciones que realizan otros profesionales sanitarios, así mismo el acceso a literatura científica y apoyo en investigación por parte de direcciones de enfermería, etc. El 50\% de las participantes respondieron negativamente al preguntar si recibían apoyo y motivación por parte de la dirección de enfermería para investigar. El 43\% refiere que enfermería no recibe los mismos apoyos externos (becas, premios, publicaciones) que otros profesionales sanitarios para investigar. El $53 \%$ considera que tiene acceso y el $30 \%$ no tiene acceso a estas fuentes. El 48\% considera que para investigar se necesitan recursos económicos difíciles de conseguir, el $50 \%$ se sienten discriminadas con respecto a otros profesionales en cuanto a contar con los mismos apoyos económicos para investigar. El $54 \%$ afirma no tener acceso a materiales, en cuanto a literatura científica, el 56\% refieren que no tienen acceso a ella en su puesto de trabajo, el $46 \%$ considera que el resto del equipo multidisciplinario colaboraría en un proyecto de investigación en enfermería.

\section{Bloque 4: Desarrollo profesional}

Este bloque se relaciona con temas profesionales como la colaboración de enfermería con otros profesionales en trabajos de investigación, o si la investigación era una función propia o delegada.

El 78\% refiere que investigar es una función de la actividad profesional, el $85 \%$ está de acuerdo en que como profesionales de enfermería tienen autoridad para decidir sobre los cuidados del paciente, el $64 \%$ está en desacuerdo en que las funciones de enfermería son fundamentalmente prácticas y no precisan incluir investigación, el 79\% estarían dispuestas a colaborar con otros profesionales en trabajos de investigación. En los mismos términos la mayoría (90\%) expresa que la investigación aporta beneficios a la práctica profesional y el $68 \%$ considera que necesita investigar para mejorar en su trabajo; el 78\% manifiesta que la investigación es una función que no debe ser delegada por otros profesionales. El $75 \%$ enfatiza que no es difícil encontrar campos interesantes en los cuales investigar; y en este sentido, el $76 \%$ considera que investigar mejora el reconocimiento profesional. También hay que destacar que, el $64 \%$ refiere que enfermería es una profesión orientada hacia la investigación.

\section{Bloque 5: Motivaciones}

En este último bloque se incluyen aspectos relacionados con la importancia de la motivación para investigar, el reconocimiento profesional, lo concerniente a alcanzar un grado en la carrera profesional y otras recompensas de tipo económico. El 73\% refiere que le gustaría participar en un trabajo de investigación y el $79 \%$ creen que tener motivación en el trabajo las anima a investigar. Por otra parte, el 63\% se siente capaz de liderar un trabajo de investigación, el $75 \%$ considera que alcanzar un grado superior en la carrera profesional las motiva a investigar, el $86 \%$ consideran que una recompensa económica aumentaría su interés por investigar. El 73\% refieren que merece la pena investigar para mejorar el currículum y ser más competitivas y el $76 \%$ les interesan los trabajos de investigación que se desarrollan en su centro de trabajo.

El nivel de confiabilidad del instrumento se evaluó a través del alfa de Cronbach, el cual obtuvo un puntaje de 0,87 , considerándose un nivel alto.

Para establecer la relación entre las variables se realizó inicialmente el análisis factorial exploratorio, el cual no mostró factores fuertes; por lo tanto, se realizó la rotación de Varimax y esta mostró quince factores fuertemente agrupados (Tabla 2). En este sentido, se podría plantear un ajuste al instrumento para facilitar el uso del mismo en las diferentes instituciones de salud y realizar seguimiento periódico a los profesionales de enfermería para detectar el desarrollo de la investigación.

Después de calcular la prueba $\mathrm{X}^{2}$ se describen las correlaciones más fuertes encontradas entre los datos sociodemográficos y los ítems de la dimensión que hacen referencia a la motivación de los profesionales de enfermería hacia la investigación (Tabla 3).

El estado civil, soltera, presentó mayor relación en cuanto a considerar que publicar un trabajo de investigación está reconocido profesionalmente. Así mismo, el estado civil presenta fuertes correlaciones con: el gusto en participar en un trabajo de investigación, el sentirse capaz de liderar un trabajo de investigación, una recompensa económica aumentaría el interés por la investigación, consideran que vale la pena investigar para aumentar la puntuación de su currículum, y se interesan en las investigaciones que se desarrollan en su centro de trabajo. 
El estado civil, soltera, presentó mayor relación en cuanto a considerar que publicar un trabajo de investigación está reconocido profesionalmente. Así mismo, el estado civil presenta fuertes correlaciones con: el gusto en participar en un trabajo de investigación, el sentirse capaz de liderar un trabajo de investigación, una recompensa económica aumentaría el interés por la investigación, consideran que vale la pena investigar para aumentar la puntuación de su currículum, y se interesan en las investigaciones que se desarrollan en su centro de trabajo.

El nivel educativo de pregrado presentó relación con: estar motivada en su trabajo la anima a investigar, considerar que vale la pena investigar para aumentar la puntuación de su currículum y el interesarse en las investigaciones que se desarrollan en su centro de trabajo.

El tener personas a su cargo se relaciona con: considerar que alcanzar un grado superior en su carrera profesional es un estímulo para investigar; además que una recompensa económica aumentaría su interés por la investigación, y el interesarse en las investigaciones que se desarrollan en su centro de trabajo.

El tiempo de antigüedad en el servicio de 1-5 años se relaciona con: el interesarse en las investigaciones que se desarrollan en su centro de trabajo, y la edad promedio de 31 años estuvo relacionada con: el estar motivada en su trabajo, la anima a investigar y sentirse capaz de liderar un trabajo de investigación.

Finalmente, las variables que mostraron significancia estadística fueron:

Variables cualitativas: Nivel educativo con las variables "Me siento capaz de liderar un trabajo de investigación", y "Alcanzar un grado superior en mi carrera profesional es un aliciente (estímulo) para investigar" con una $\mathrm{p}<0,05$, lo que indica que la respuesta o nivel de motivación reportado fue diferente por los grupos de nivel educativo.

Variables cuantitativas: Tiempo de antigüedad y "Estar motivada en mi trabajo me anima a investigar” presentaron un rho -0,2768 con una p de 0,0129. Tiempo de antigüedad y "Alcanzar un grado superior en mi carrera profesional es un estímulo para investigar" presentaron un rho -0,2357 con una p de 0,0353 . Es decir, tienen una relación inversamente proporcional, dado que a mayor antigüedad menos están motivadas en el trabajo para investigar y alcanzar un grado superior en la carrera profesional es un estímulo para investigar.

Tabla 2. Factores

\begin{tabular}{|c|c|c|}
\hline Dimensión & Factor & Valor $\mathrm{p}$ \\
\hline \multirow[t]{3}{*}{ Bloque 1} & Mi vida personal se verá afectada negativamente si realizo un trabajo de investigación. & 0,72 \\
\hline & $\begin{array}{l}\text { Hacer un trabajo de investigación durante la jornada laboral entorpecería el desarrollo de mis fun- } \\
\text { ciones. }\end{array}$ & 0,84 \\
\hline & La distribución de la jornada laboral por turnos dificulta dedicarle tiempo a la investigación. & 0,70 \\
\hline \multirow[t]{3}{*}{ Bloque 2} & No existe suficiente formación para la investigación enfermera. & 0,78 \\
\hline & El conocimiento de otros idiomas facilita la investigación. & 0,83 \\
\hline & $\begin{array}{l}\text { Enfermería está igual de capacitada que otros profesionales sanitarios para realizar estudios de in- } \\
\text { vestigación. }\end{array}$ & 0,74 \\
\hline \multirow[t]{3}{*}{ Bloque 3} & $\begin{array}{l}\text { En mi trabajo, el resto del equipo multidisciplinar colaboraría en un proyecto de investigación en } \\
\text { enfermería. }\end{array}$ & 0,82 \\
\hline & $\begin{array}{l}\text { En mi puesto de trabajo tengo acceso a recursos materiales (papel, ordenador, programas informá- } \\
\text { ticos...) para investigar. }\end{array}$ & 0,82 \\
\hline & Las funciones de enfermería son fundamentalmente prácticas y no precisan incluir investigación. & 0,81 \\
\hline \multirow[t]{2}{*}{ Bloque 4} & No necesito investigar para mejorar en mi trabajo. & 0,81 \\
\hline & Investigar mejora el reconocimiento de la profesión enfermera. & 0,76 \\
\hline \multirow[t]{2}{*}{ Bloque 5} & Me gustaría participar en un trabajo de investigación. & 0,85 \\
\hline & Me siento capaz de liderar un trabajo de investigación. & 0,72 \\
\hline
\end{tabular}


Tabla 3. Variables de relación

\begin{tabular}{|c|c|c|}
\hline Preguntas & Variable & Valor prueba $\mathrm{X}^{2}$ \\
\hline Me gustaría participar en un trabajo de investigación. & $\begin{array}{l}\text { Estado civil } \\
\text {-Solteras } \\
\text {-Casadas }\end{array}$ & 0,909 \\
\hline Estar motivado en mi trabajo me anima a investigar. & $\begin{array}{l}\text { Nivel educativo } \\
\text {-Pregrado }\end{array}$ & 0,545 \\
\hline Me siento capaz de liderar un trabajo de investigación. & $\begin{array}{l}\text { Estado civil } \\
\text {-Solteras } \\
\text {-Casadas }\end{array}$ & 0,519 \\
\hline Alcanzar un grado superior en mi carrera profesional es un aliciente (estímulo) para investigar. & $\begin{array}{c}\text { Personas a cargo } \\
\text {-Sí }\end{array}$ & 0,536 \\
\hline Una recompensa económica aumentaría el interés por la investigación. & $\begin{array}{l}\text { Estado civil } \\
\text {-Solteras } \\
\text {-Casadas }\end{array}$ & 0,956 \\
\hline Una recompensa económica aumentaría el interés por la investigación. & $\begin{array}{c}\text { Personas a cargo } \\
\text {-Sí }\end{array}$ & 0,607 \\
\hline Publicar un trabajo de investigación no está reconocido profesionalmente. & $\begin{array}{c}\text { Estado civil } \\
\text {-Solteras }\end{array}$ & 0,633 \\
\hline No merece la pena investigar para aumentar la puntuación de mi curriculum. & $\begin{array}{l}\text { Estado civil } \\
\text {-Solteras } \\
\text {-Casadas }\end{array}$ & 0,584 \\
\hline No merece la pena investigar para aumentar la puntuación de mi curriculum. & $\begin{array}{l}\text { Nivel educativo } \\
\text {-Pregrado }\end{array}$ & 0,531 \\
\hline Me interesan los trabajos de investigación que se desarrollan en mi centro de trabajo. & $\begin{array}{l}\text { Estado civil } \\
\text {-Solteras } \\
\text {-Casadas }\end{array}$ & 0,808 \\
\hline Me interesan los trabajos de investigación que se desarrollan en mi centro de trabajo. & $\begin{array}{c}\text { Personas a cargo } \\
\text {-Sí }\end{array}$ & 0,556 \\
\hline Me interesan los trabajos de investigación que se desarrollan en mi centro de trabajo. & $\begin{array}{l}\text { Nivel educativo } \\
\text {-Pregrado }\end{array}$ & 0,896 \\
\hline Estar motivado en mi trabajo me anima a investigar. & $\begin{array}{c}\text { Edad } \\
\text {-Promedio } 31\end{array}$ & 0,588 \\
\hline Me siento capaz de liderar un trabajo de investigación & $\begin{array}{c}\text { Edad } \\
\text {-Promedio } 31\end{array}$ & 0,798 \\
\hline Me interesan los trabajos de investigación que se desarrollan en mi centro de trabajo. & $\begin{array}{c}\text { Tiempo antigüe- } \\
\text { dad en servicio } \\
1-5 \text { años }\end{array}$ & 0,953 \\
\hline
\end{tabular}

\section{DISCUSIÓN}

La participación global en este estudio fue del 53\% $(\mathrm{n}=80)$ de enfermeras, del total de 150 profesionales de enfermería de una institución de salud de tercer nivel de atención. Este dato es importante porque es un referente de comparación con los hallazgos encontrados en otro estudio en España ${ }^{12}$ cuya participación global de enfermeras fue $41 \%$, y en el que se enfatiza sobre la importancia del conocer la actitud hacia la actividad investigadora por parte de los profesionales de enfermería para promover más la investigación. Además, las participantes pertenecían a distintos servicios de la institución, similar a otro estudio en Turquia ${ }^{13}$, lo cual favorece tener una percepción general de las enfermeras de las diferentes áreas asistenciales.

La mayoría de las enfermeras no tienen experiencia en investigación a diferencia de lo encontrado en un estudio en España ${ }^{9}$, en el que un alto porcentaje había participado en más de una investigación.

En general, las participantes en este estudio consideran que investigar contribuye al desarrollo de la profesión y permite mejorar la calidad asistencial, lo anterior concuerda con una actitud positiva hacia la investigación por parte de enfermeras clínicas encontrado en 
otros estudios por Akerjordet y colaboradores ${ }^{5}$ y Bayik y colaboradores ${ }^{13}$.

La mayoría de las enfermeras expresaron que investigar es una actividad necesaria y que es función de la actividad profesional, además, en general están en desacuerdo en que las funciones de enfermería son fundamentalmente prácticas y no precisan incluir investigación. Contrario a un estudio en España ${ }^{12}$ en el cual se evidenció que tan solo el $23 \%$ lo considera una actividad necesaria.

En esta investigación se encontró que muy pocas enfermeras han publicado, al igual que en otros estudios por Díaz y colaboradores ${ }^{12}$ y Cadena y colaboradores $^{14}$ y lo expuesto por otros autores ${ }^{2}$ quienes encontraron un reducido número de publicaciones de las enfermeras asistenciales.

A la mayoría de enfermeras, en este estudio, les resulta difícil investigar fuera de su horario laboral, no disponen de tiempo durante su jornada laboral para investigar y consideran que investigar en su horario laboral entorpecería el resto de sus funciones. Otro problema importante para las participantes es la elevada carga asistencial, que obstaculiza la investigación durante su jornada laboral; por lo que manifiestan que se debe destinar una parte de la jornada laboral para trabajar en estudios de investigación. Estos resultados concuerdan con lo encontrado por Cepeda y colaboradores ${ }^{9}$.

Las participantes consideran que su vida personal no se verá afectada si realizan un trabajo de investigación y que sus obligaciones fuera del horario laboral no le suponen un obstáculo para investigar. Contrario al estudio en Perú ${ }^{15}$ en el que los resultados obtenidos indicaron que las enfermeras muestran una actitud desfavorable hacia la investigación por factores personales.

En relación con conocimientos y preparación para la investigación, $46 \%$ considera que tiene suficientes conocimientos para realizar un proyecto de investigación, un porcentaje mayor se encontró en otro estudio por Díaz et al. ${ }^{12}$ en el que $80 \%$ considera que no tiene la formación suficiente para investigar.

Se destaca la importancia de la lectura continua, el conocimiento de nuevas tecnologías y además se expresa que no existe formación en investigación suficiente y que se deberían realizar cursos de posgrado relacionados con la materia. Como lo expuesto por Vallejos ${ }^{16}$ en su estudio.

Para la mayoría de participantes en esta investigación, en los planes de estudio se debería incluir formación en investigación. Esto concuerda con lo que menciona Aguirre ${ }^{17}$ que la formación en investigación debe promoverse desde el pregrado, dada su importancia para el profesional de enfermería. Además, la generalidad de enfermeras consideró que, el saber otros idiomas facilita investigar, como lo encontrado en otro estudio ${ }^{6}$ donde se considera una barrera el desconocimiento del idioma inglés.

La mayoría de las participantes respondieron que falta apoyo, recursos económicos y materiales para hacer investigación, además que se sienten discriminadas con respecto a otros profesionales en cuanto a contar con los mismos apoyos económicos para investigar, similar como lo encontrado en otro estudio en España ${ }^{12}$. En relación con los recursos materiales, fuentes de información y el acceso a literatura científica, la mayoría afirma no tener acceso a ellos. Similar a otros estudios ${ }^{12,18}$ en los que se desconocen la existencia de recursos institucionales para la investigación.

En este estudio, la mayoría de las participantes consideran que la investigación aporta beneficios para la práctica profesional, que es necesario investigar para mejorar su trabajo, el reconocimiento profesional, que no debe ser delegada la investigación a otros profesionales y que enfermería es una profesión orientada a la investigación. Al contrario, que lo encontrado en un estudio $^{12}$ en el que $77 \%$ menciona que no es imprescindible investigar para el desarrollo y el avance de la profesión.

En general, la mayoría de las participantes refiere que enfermería está igual de capacitada que otros profesionales sanitarios para realizar un estudio de investigación. Además, que estarían dispuestos a trabajar con otros profesionales en investigación y que el resto del equipo multidisciplinar colaboraría en un proyecto de investigación en enfermería. En general, las enfermeras enfatizan que no es difícil encontrar campos interesantes en los cuales investigar; que les gustaría participar en investigación, y que les intere- 
san los trabajos de investigación que se desarrollan en su centro de trabajo y que ayuden a decidir los cuidados del paciente. Además, una recompensa económica aumentaría su interés por investigar y creen que estar motivadas en el trabajo les anima a investigar. Lo anterior, evidencia que las enfermeras en este estudio mostraron motivación personal hacia la investigación, a diferencia de otro estudio ${ }^{12}$ que consideran como obstáculos los problemas de actitud del personal de salud para investigar por respuestas como "no sé", "no tengo tiempo" y "no puedo y no quiero", por lo cual invitan a conocer la actitud hacia la actividad investigadora para hacer una adecuada promoción de la investigación. En un estudio en Canadá ${ }^{19}$ en enfermeras del área de ortopedia se encontró que tenían interés y motivación para investigar, pero que esta motivación se inhibe por falta de apoyo y tiempo en su trabajo.

Uno de los principales resultados encontrados fue la relación entre el nivel educativo, liderar trabajos de investigación y alcanzar un grado superior. En esta investigación se encontró que a mayor nivel educativo y considerar que alcanzar un grado superior en la carrera profesional las motiva a investigar y sentirse capaz de liderar trabajos de investigación. Además, la mayoría de las enfermeras refieren que merece la pena investigar para mejorar el currículum y ser más competitiva, lo cual, concuerda con otro estudio realizado por Cadena y colaboradores ${ }^{14}$ donde se expone que el desarrollo de la investigación va de la mano con el avance en la educación. También, en otro estudio en Singapur ${ }^{20}$ se encontró que enfermeras más jóvenes y con un nivel educativo más alto participan más fácilmente en investigación, se motivan a liderar proyectos, utilizan más la tecnología y se sintieron más competentes. En otro estudio de Bayik y colaboradores ${ }^{13}$ no se halló una diferencia estadísticamente significativa entre hacer parte de una investigación y grupos de edad y estado civil, pero sí se encontró una diferencia significativa entre hacer parte de una investigación y el nivel educativo. En el estudio multicéntrico de Siedlecki y Albert $^{21}$ se encontró que a mayor nivel educativo tienen más conocimiento en investigación.

También como un resultado importante fue el tiempo de antigüedad y estar motivadas en el trabajo anima a investigar y alcanzar un grado superior. En un estudio descriptivo ${ }^{20}$ se encontró que el tiempo de antigüedad no influyó en el valor e interés que la enfermera le da a la investigación, pero sí que a mayor tiempo de antigüedad han tenido más experiencia investigativa, $y$ a menor tiempo de antigüedad utilizan más la tecnología y se sienten más competentes para investigar.

En general, los resultados de este estudio en las enfermeras de Colombia concuerdan con lo encontrado en enfermeras de México ${ }^{14}$ y de España ${ }^{9}$, la carga laboral, el poco tiempo disponible durante y fuera del horario laboral y las obligaciones familiares afectan negativamente para realizar una investigación, sin embargo, sí están interesadas en investigar, si se otorga el tiempo en la jornada laboral. Las enfermeras de Perú ${ }^{15}$ consideran que las barreras personales son las principales. Las enfermeras turcas ${ }^{6}$ consideran que es la falta de tiempo y la dificultad para el inglés. En un estudio multicéntrico ${ }^{20}$ con enfermeras de Canadá, publicado recientemente, identificó la brecha persistente entre la adquisición del conocimiento y su aplicación en entornos clínicos, por la baja investigación en enfermeras asistenciales. Además, encontraron como facilitadores para la investigación el ser enfermeras jóvenes con alto nivel educativo y el apoyo en su lugar de trabajo. En otro estudio multicéntrico con enfermeras de Singapour ${ }^{21}$, los resultados mostraron la necesidad de programas de entrenamiento continuo, con el fin de prepararlas mejor para la práctica basada en la evidencia. En un estudio con enfermeras chinas ${ }^{8}$ se encontraron como barreras para la investigación la falta de tiempo, de financiación y el lenguaje y como facilitador el aumento en los conocimientos.

Un estudio cualitativo en enfermeras de Estados Unidos $^{22}$ encontró que la conducta hacia la investigación por parte de enfermeras clínicas, influye en su rol en la generación de conocimiento, y que la presencia de condiciones de apoyo, como un mentor de investigación favorece la investigación. Las enfermeras clínicas pueden llevar a cabo investigaciones, y lo hacen, a pesar de las limitaciones debidas a la falta de tiempo, dinero y conocimiento, si tienen acceso a mentores de investigación y si están en un entorno de apoyo a la investigación. En un estudio cualitativo con enfermeras del Reino Unido ${ }^{23}$ publicado recientemente se encontró que las enfermeras expresaron dificultades para poder investigar en el área clínica y que los sacrificios personales eran comunes y consideraron que estos no fueron reconocidos por los 
gerentes. Que la investigación necesita sistemas de apoyo más estructurados y el reconocimiento del esfuerzo que hace la enfermera para investigar.

En otro estudio de Akerjordet et al., los resultados mostraron que las enfermeras clínicas enfatizaron que la falta de tiempo designado, el interés y el conocimiento constituyeron las barreras de investigación más importantes ${ }^{5}$. Así mismo, en una revisión sistemática ${ }^{23}$ se encontró que las enfermeras consideran la investigación como necesaria y esencial para la profesión de enfermería y el apoyo institucional fue percibido como el principal facilitador de participación en actividades de investigación. El segundo facilitador más importante fue la actitud y la motivación de las enfermeras.

Se señala que existe necesidad de mayor formación y motivación hacia la investigación, especialmente ahora que la tecnología, la comunicación y la evidencia científica son parte importante de la globalización del cuidado humano y de la sustentación para desarrollar una práctica basada en un conocimiento más profundo y así poder llenar los vacíos que deja el centrarse en el cuidado desde la perspectiva biológica ${ }^{2}$.

En conclusión, se evidencia que uno de los factores asociados con el desarrollo de la investigación en enfermería en el área clínica, es que a mayor nivel educativo del profesional de enfermería, mayor motivación a investigar y de sentirse capaz de liderar trabajos de investigación. El tiempo de antigüedad en la institución es también un factor, dado que a mayor antigüedad, menos están motivados a investigar y otro factor importante es la motivación que tengan en el trabajo para investigar. Durante las últimas décadas la demanda del conocimiento y la práctica clínica basada en la evidencia ha aumentado y se requiere una colaboración entre enfermería a nivel académico y clínico, para impulsar la investigación que le sea útil a la práctica. Así mismo, es necesario que las instituciones de salud favorezcan la investigación y se desarrolle una cultura para fortalecer la capacidad de investigación en los profesionales de enfermería clínicos, y así generar resultados que mejoren el cuidado de los pacientes.

Finalmente, dentro de las limitaciones de este estudio se encuentra que el muestreo no fue aleatorio, dada la baja respuesta de las enfermeras al diligenciar el cuestionario, sin embargo, participaron de todos los servicios para tener una visión global.

\section{REFERENCIAS}

1. Varela MD, Sanjurjo MD, Blanco FJ. La investigación en enfermería. Rol de la enfermería Rev. Asoc. Esp. Enferm. Urol. 2012;121:19-21.

2. Díaz LP, Bueno LS, Mejías Rojas ME, López L. Investigación de enfermería: una caracterización en dos publicaciones latinoamericanas. Av en Enfermería. 2011;29(2): 285-93.

3. Diario Oficial No. 42.710, del 5 de febrero de 1996, Ley 266 de 1996, [acceso 01 julio 2017] Disponible en: http://www.mineducacion.gov.co/1759/articles-105002_ archivo_pdf.pdf.

4. Corchon S, Potillo MC, Watson R, Saracíbar M. Nursing research capacity building in a Spanish hospital: an intervention study. J Clin Nurs. 2011;20:2479-2489. DOI: 10.1111/j.1365-2702.2011.03744.x.

5. Akerjordet K, Lode K, Severinsson E. Clinical nurses' attitudes towards research, management and organisational resources in a university hospital: part 1. J Nurs Manag. 2012;20:814-823. DOI: 10.1111/j.1365-2834.2012.01477.x.

6. Kocaman G, Seren S, Lash A, Kurt S, Bengu N, Barriers to research utilisation by staff nurses in a university hospital. Journal of Clinical Nursing. 2010;19:1908-1918. DOI: 10.1111/j.1365-2702.2009.03032.x.

7. Wangensteen S, Johansson I, Bjorkstrom M, Nordstrom G. Research utilisation and critical thinking among newly graduated nurses: predictors for research use. A quantitative cross-sectional study. J Clin Nurs. 2011;20:24362447. DOI: $10.1111 /$ j.1365-2702.2010.03629.x.

8. Wang LP, Jiang XL, Wang L, Wang GR, Bai YJ. Barriers to and Facilitators of Research Utilization: A Survey of Registered Nurses in China. PLoS ONE. 2013;8,11: e81908. DOI: 10.1371/journal.pone.0081908.

9. Cepeda JM. Validación de un cuestionario sobre la motivación hacia la investigación en enfermería. Rev. Enferm. CyL. 2009;1(2):30-9.

10. Ministerio de salud de la República de Colombia. Resolución número 8430 de 1993, [acceso 01 julio 2017] Disponible en: https://www.minsalud.gov.co/sites/ rid/Lists/BibliotecaDigital/RIDE/DE/DIJ/RESOLUCION-8430-DE-1993.PDF. 
11. Consejo de Organizaciones Internacionales de las Ciencias Médicas (CIOMS). Pautas éticas internacionales para la investigación biomédica en seres humanos. Ginebra 2002. [acceso 01 julio 2017] Disponible en: http://www. ub.edu/rceue/archivos/Pautas_Eticas_Internac.pdf.

12. Díaz M, Leal CM, Jiménez JM, Maya B. Producción científica de los profesionales de enfermería en un hospital de tercer nivel. Enferm Clin. 2004;14:263-8. DOI: 10.1016/ S1130-8621(04)73896-6.

13. Bayik A, Ardaha M, Odak S. An Evaluation of Nurses' Opinions towards Organizational Research Culture. Balkan Journal of Health Science. 2015;3(4);74-82.

14. Cadena JC, Olvera SS, Teniza D, López L. Factores que influyen en el profesional de enfermería de una institución de tercer nivel para desarrollar investigación. 2014;48:28-37.

15. Choque LR. Factores personales e institucionales que intervienen en la actitud de la enfermera hacia la investigación en el hospital nivel III. Universidad Nacional Jorge Basadre Grohmann. 2013. [acceso 8 julio 2017] Disponible en: http://redi.unjbg.edu.pe/handle/UNJBG/300.

16. Vallejos VJ. Motivación hacia la investigación que poseen los profesionales de enfermería en dos hospitales públicos de Lima Este, 2015 [acceso 8 julio 2017] Disponible en: http://repositorio.upeu.edu.pe/bitstream/handle/UPEU/563/V\%C3\%ADctor_Tesis_bachiller_2016.pdf?sequen$\mathrm{ce}=1$ \&isAllowed $=y$ Aguirre D. La investigación en enfermería en América Latina 2000-2010. Revista Habanera de Ciencias Médicas. 2011;10,3:396-409.

17. Higgins I, Parker V, Keatinge D, Giles M, Winskill R, Guest E, et al. Doing clinical research: the challenges and benefits. Contemp Nurse. 2010;(35),2:171-81. DOI: 10.5172/conu.2010.35.2.171.
18. Berthelsen CB, Hølge-Hazelton B. Orthopaedic nurses' attitudes towards clinical nursing research - A cross-sectional survey. Int J Orthop Trauma Nurs. 2015;(19),2:7484. DOI:10.1016/j.ijotn.2014.10.004.

19. Robichaud S. New Brunswick nurses' views on nursing research, and factors influencing their research activities in clinical practice. Nurs Health Sci. 2016;(18),2:246-55. DOI: $10.1111 /$ nhs. 12261.

20. Shin Yuh Ang, RN, MBA, Xui LingKwan, RN, BN, Fazila Abu BakarAloweni, RN, MSc, Ai Tee Aw, RN, MNR, Ai Meng Tan, RN, MHA, et al. A Descriptive Study on Registered Nurses' Continuing Educational Needs in Research from Three Different Health Care Institutes in Singapore. Proceedings of Singapore Healthcare. 2015;(24),2:72-79. DOI: $10.1177 / 201010581502400202$.

21. Siedlecki S. Albert N. Research-active clinical nurses: against all odds. J Clin Nurs. 2016;26:766-773. DOI: 10.1111/jocn.13523.

22. Woodward V, Webb C, Prowse M. The perceptions and experiences of nurses undertaking research in the clinical setting. Journal of research in Nursing. 2016;(12),3: 227244. DOI:10.1177/1744987107077398.

23. Lode k, Erik E, Salmela S, Lise A, Severinsson E. Clinical Nurses' Research Capacity Building in Practice: A Systematic Review. Journal of Nursing. 2015;5:664-677. DOI: 10.4236/ojn.2015.57070. 